\title{
Design of a paper-based reconfigurable frequency selective surface structure
}

\author{
Sung-Sil Cho and Ic-Pyo Honga) \\ Department of Information \& Communication Engineering, \\ Kongju National University, 1223-24 Cheonandae-ro, Cheonan, 31080, Korea \\ a)iphong@kongju.ac.kr
}

\begin{abstract}
In this study, a paper-based reconfigurable frequency selective surface (FSS) that can be applied as wallpaper inside buildings to control the indoor spectrum was designed using the ink-jet printing method. The designed paper-based FSS uses a PIN diode to block $5 \mathrm{GHz}$ band signals in the OFF condition and to transmit the signals in the ON condition, offering frequency characteristics stable to polarization and incident angles. To verify the operation of the designed FSS, the FSS was printed on a $0.2 \mathrm{~mm}$ thick paper using the ink-jet printing method by attaching a PIN diode using a conductive adhesive to manufacture the FSS with a switching function. It was confirmed that the measured frequency transmission characteristics according to the switching operation corresponded to the calculated results, and stability was exhibited for polarization and incident angles.
\end{abstract}

Keywords: reconfigurable frequency selective surface, ink-jet printing Classification: Microwave and millimeter-wave devices, circuits, and modules

\section{References}

[1] L. Subrt, et al:: "Controlling coverage for indoor wireless networks using metalized active FSS walls," APCC (2013) 496 (DOI: 10.1109/APCC.2013. 6765998).

[2] P. S. Taylor, et al.: "An active annular ring frequency selective surface," IEEE Trans. Antennas Propag. 59 (2011) 3265 (DOI: 10.1109/TAP.2011.2161555).

[3] B. Sanz-Izquierdo and E. A. Parker: "Dual polarized reconfigurable frequency selective surfaces," IEEE Trans. Antennas Propag. 62 (2013) 764 (DOI: 10. 1109/TAP.2013.2292056).

[4] A. A. Dewani, et al:: "Screen printed frequency selective surfaces for room isolation in buildings," IWAT (2014) 208 (DOI: 10.1109/IWAT.2014.6958640).

[5] B. M. Turki, et al.: "Study of clusters of defects in low-cost digitally fabricated frequency selective surface," EuCAP (2014) 779 (DOI: 10.1109/EuCAP.2014. 6901882).

[6] Y. Kawahara, et al:: "Instant inkjet circuits: lab-based inkjet printing to support rapid prototyping of UbiComp devices," ACM (2013) 363 (DOI: 10.1145/ 2493432.2493486).

[7] B. S. Cook and A. Shamim: "Utilizing wideband AMC structures for high-gain inkjet-printed antennas on lossy paper substrate," IEEE Antennas Wireless Propag. Lett. 12 (2013) 76 (DOI: 10.1109/LAWP.2013.2240251). 
[8] R. Sivasamy, et al.: "A low-profile paper substrate-based dual-band FSS for GSM shielding,” IEEE Trans. Electromagn. Compat. 58 (2016) 611 (DOI: 10. 1109/TEMC.2015.2498398).

[9] L. B. Wang, et al.: "Ultrathin and flexible screen-printed metasurfaces for EMI shielding applications," IEEE Trans. Electromagn. Compat. 53 (2011) 700 (DOI: 10.1109/TEMC.2011.2159509).

[10] N. Qasem and R. Seager: "Indoor band pass frequency selective wall paper equivalent circuit and ways to enhance wireless signal," LAPC (2011) 1 (DOI: 10.1109/LAPC.2011.6114081).

[11] I. Hong: "Paper-based frequency selective surface for stable angle of incidence," IEICE Electron. Express 12 (2015) 20150185 (DOI: 10.1587/ elex.12.20150185).

[12] H. Li, et al:: "A novel active frequency selective surface with switching performance for $2.45 \mathrm{GHz}$ WLAN band," APMC (2015) 1 (DOI: 10.1109 / APMC.2015.7411716).

\section{Introduction}

The number of users who operate wireless devices in indoor spaces is increasing owing to the rapid development of wireless communication technology. Accordingly, numerous wireless signals exist in indoor spaces, causing problems such as unhealthy influences harmful to the human body or the deterioration of communication performance owing to interference between signals. A reconfigurable frequency selective surface (FSS) was proposed as a method to adjust the propagation environment to serve the users' objective by actively blocking or transmitting particular signals in indoor spaces, as well as solving the abovementioned problems $[1,2,3]$. However, the proposed FSS is being manufactured in PCB form by using chemical methods of etching copper on substrates. This method has disadvantages such as complex processes and high manufacturing cost. Thus, it is difficult to apply this method to actual indoor spaces [4, 5].

Conductive ink such as silver nanoparticle ink is used in ink-jet printing, which allows for quick and inexpensive manufacturing even for complex structures $[6,7,8]$. In addition, paper or transparent films that are inexpensive with flexible features can be used in wallpaper applications $[4,5]$. Therefore, various studies are being conducted to improve communication performance by enhancing wireless signal intensity, reducing interference between wireless signals such as those from WLANs, and blocking electromagnetic interference (EMI) in indoor spaces [9] by manufacturing an FSS using the ink-jet printing method [10, 11]. However, only particular frequencies are blocked, preventing the establishment of various propagation environments.

In this paper, a paper-based FSS that can be applied as wallpaper by using the ink-jet printing method and can actively block or transmit particular frequencies using a PIN diode was designed. In addition, the performance of the designed structure was verified through manufacturing and measurement tests. 


\section{Design and simulation}

Changes in propagation generally occur more frequently in indoor spaces than in outdoor spaces, so propagation environments with different incident angles and polarization exist [12]. Therefore, an FSS that is stable to incident angles and polarization and is suitable to this communication environment was designed in this study.

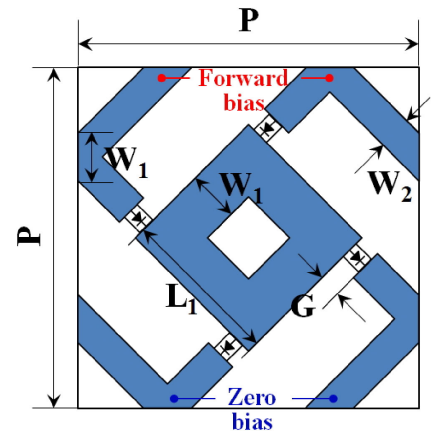

(a) Unit cell

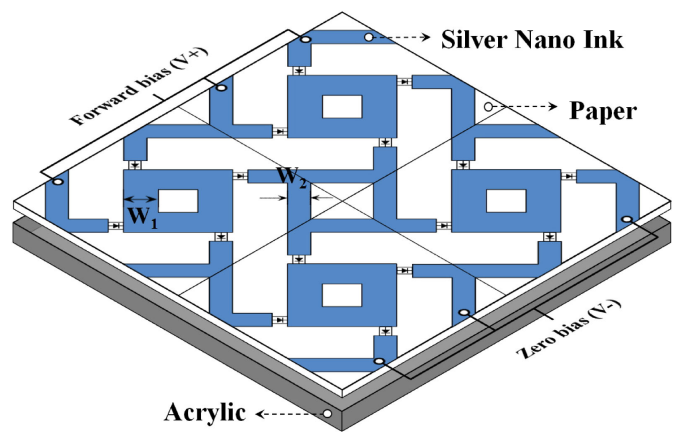

(b) Side view of unit cell array

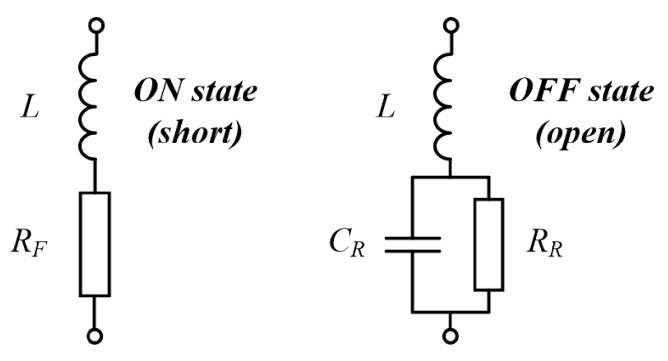

(c) Equivalent circuits of PIN diode

Fig. 1. Unit cell geometry of proposed reconfigurable FSS and equivalent circuit of PIN diode

The unit structure of the FSS is shown in Fig. 1(a). The period of the unit structure is $P=17 \mathrm{~mm}$. Regarding the design variables of the unit structure, the outer diameter of the rectangle loop was $\mathrm{L}_{1}=7.9 \mathrm{~mm}$, the width of the rectangle loop was $\mathrm{W}_{1}=2.5 \mathrm{~mm}$, the width of the four bias lines of the PIN diode was $\mathrm{W}_{2}=\mathrm{W}_{1} / \sqrt{2}$ considering the surface resistance and the space between the rectangle loop and bias line was $\mathrm{G}=1.1 \mathrm{~mm}$ considering the PIN diode length to design the structure to block or transmit $5 \mathrm{GHz}$ band signals. We presented $2 \times 2$ array in Fig. 1(b). When the PIN diode is OFF state, the bias lines become OPEN and there will be two different square loops for band-stop of $5 \mathrm{GHz}$ wireless signal. While the PIN diode is ON state, the bias lines are connected to the square loops and there will be grid patterns for passing the $5 \mathrm{GHz}$ signal. A substrate with permittivity of $\varepsilon_{r}=3$ was used, and paper with a loss tangent of $\delta=0.02$ and height of $0.2 \mathrm{~mm}$ was used. An acrylic panel with permittivity of $\varepsilon_{r}=2.5$, loss tangent of $\delta=0.02$, and height of $5 \mathrm{~mm}$ was used. The PIN diode can easily fall off when using only paper owing to the flexibility of the paper, and so an acrylic panel was used for the convenience of manufacturing and measuring. An SMP1345079LF PIN diode from SKYWorks Solutions Company was used in this study. The PIN diode that we used has the equivalent circuit as shown in Fig. 1(c). When the 
PIN diode is ON state, the values of equivalent circuit are $\mathrm{L}=0.7 \mathrm{nH}$ and $\mathrm{R}_{\mathrm{F}}=$ $1 \Omega$, while the PIN diode is OFF state, $L=0.7 \mathrm{nH}, \mathrm{C}_{\mathrm{R}}=0.31 \mathrm{pF}$ and $\mathrm{R}_{\mathrm{R}}=1 \mathrm{M} \Omega$ are used for simulation.

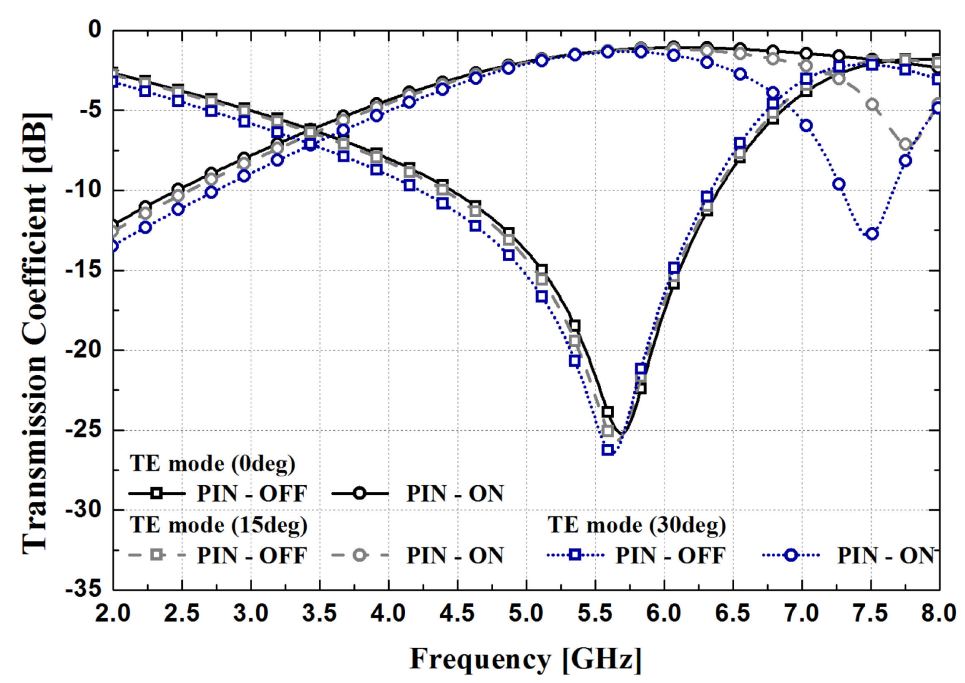

(a) TE mode

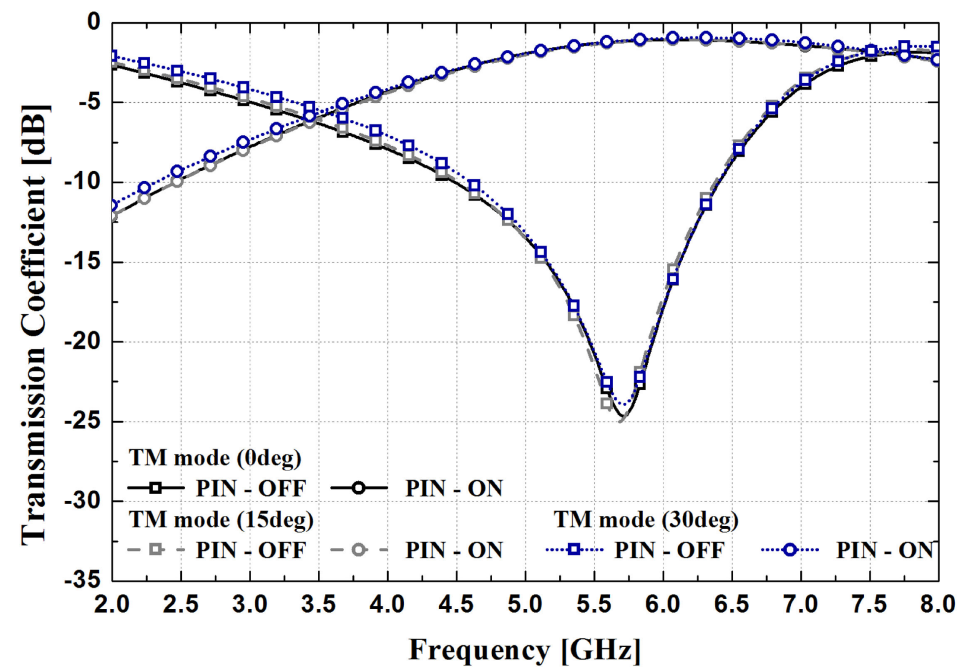

(b) TM mode

Fig. 2. Simulated transmission characteristics of the proposed reconfigurable FSS for different incident angles $\left(0^{\circ}, 15^{\circ}\right.$, and $\left.30^{\circ}\right)$

A commercial electromagnetic field analysis tool which utilizes Finite Element Method to determine the electromagnetic behavior of the structure, ANSYS HFSS, was used to investigate frequency transmission characteristics by applying the average surface resistance of the silver nano ink, $0.46 \Omega / \square$, which was obtained through the surface resistance measurement results. Floquet ports as boundary conditions were used to determine the transmission loss of the proposed FSS.

Fig. 2(a) and Fig. 2(b) show the simulation results of frequency transmission characteristics in TE mode and TM mode with an incident angle of $0^{\circ}, 15^{\circ}$, and $30^{\circ}$. When the PIN diode was in the OFF condition, a transmission coefficient of $-25.1 \mathrm{~dB}$ was shown at $5.71 \mathrm{GHz}$ in TE mode at normal incidence $\left(0^{\circ}\right)$, and $-24.5 \mathrm{~dB}$ was shown at $5.73 \mathrm{GHz}$ in $\mathrm{TM}$ mode with a resonant frequency error of 
$0.02 \mathrm{GHz}(0.35 \%)$. When the PIN diode was in the ON condition, a transmission coefficient of $-1.17 \mathrm{~dB}$ was shown in TE mode, and $-1.14 \mathrm{~dB}$ was shown in TM mode. In addition, the incident angles were changed to $15^{\circ}$ and $30^{\circ}$ to determine the frequency transmission characteristics according to changes in the incident angles. The resonant frequency error of each incident angle was $1.4 \%$ in TE mode and $0.7 \%$ in TM mode. This confirmed that the proposed structure is stable for polarization and incident angles, and that the frequency is reconfigured according to the switching of the PIN diode.

\section{Manufacturing and measurement}

A $10 \times 10$ FSS array was manufactured using the ink-jet printing method to verify the performance of the designed FSS. Silver nanoparticle ink (AN01) from the AgIC Company was used as the conductive ink, and A4-sized coated paper with a height of $177 \pm 12 \mu \mathrm{m}$ was used to manufacture the FSS. A well-known commercial ink-jet printer, MFC-J5910DW of Brother Industries Ltd, was used using a disposable piezo ink-jet cartridge [11]. General soldering could not be used owing to the vulnerability of paper to temperature, so conductive epoxy adhesive (CW2400) from the ITW Chemtronics Company was used to attach the PIN diode to the printed FSS. The final FSS is shown in Fig. 3.

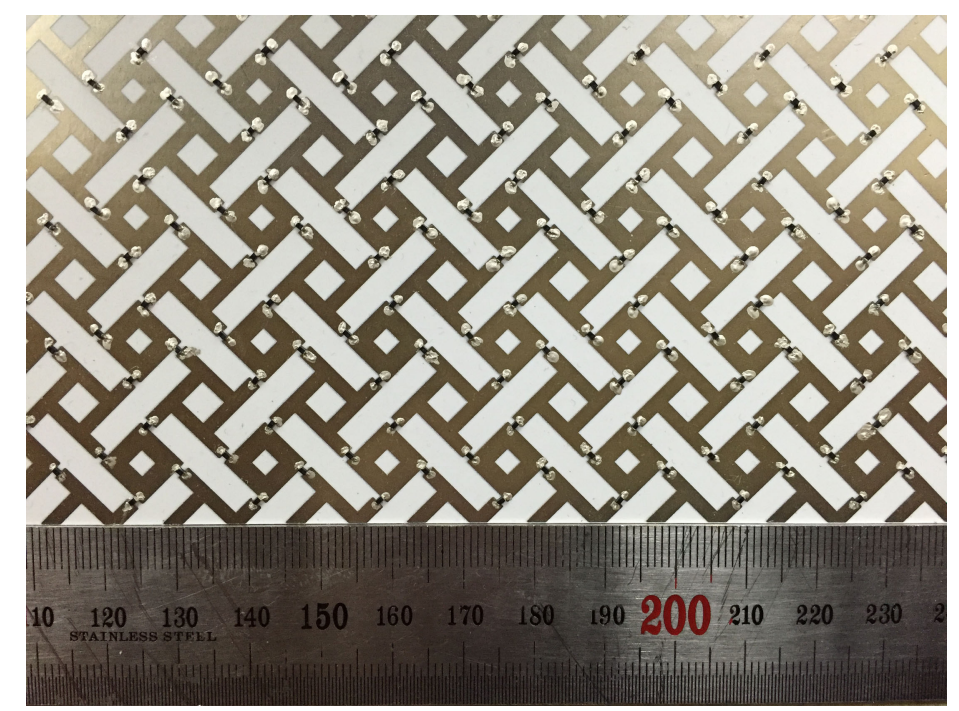

Fig. 3. Photographs of the fabricated, proposed reconfigurable FSS

We showed the equivalent circuits of the unit cell and the arrays in Fig. 4. To switch on the PIN diode, we supplied $15 \mathrm{~V}$ for bias voltage and $10 \mathrm{~mA}$ for bias current. If we don't apply any bias voltage, the PIN diode will be OFF state. The bias lines are connected to the square loop at OFF state and the grid patterns are formed for passing the $5 \mathrm{GHz}$ signal.

Free-space measurement method was used to measure the frequency transmission characteristics according to changes in incident angles and polarization. When the vertically incident signals with an incident angle of $0^{\circ}$ were used when the PIN diode was in the OFF condition $(0 \mathrm{~V})$, it was shown that signals over $90 \%$ at a resonant frequency of $5.91 \mathrm{GHz}$ were blocked in TE mode and TM mode. It is 


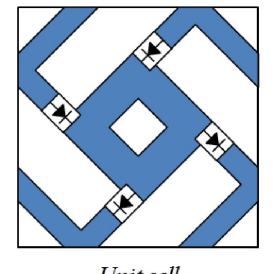

Unit cell

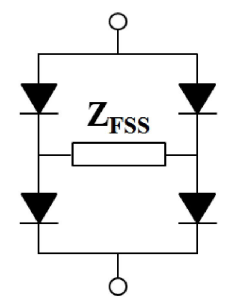

Equivalent circuit of unit cell

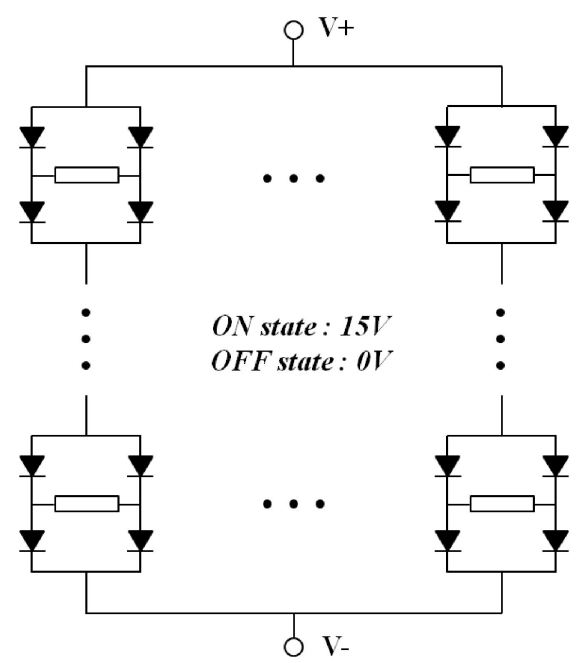

Diodes biasing scheme of reconfigurable FSS

Fig. 4. Equivalent circuits of the unit cell and the array

measured that a signal of $-1.6 \mathrm{~dB}$ is transmitted at $5.91 \mathrm{GHz}$ in the $\mathrm{ON}$ condition $(15 \mathrm{~V})$ by applying DC power to the PIN diode.

Fig. 5(a) and 5(b) show the measurements of frequency transmission characteristics according to changes in the incident angles. A resonant frequency error of

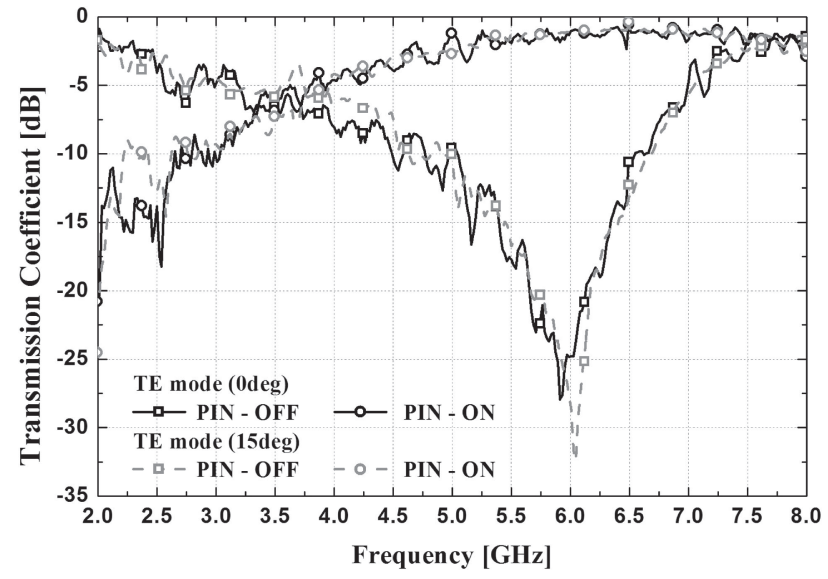

(a) TE mode

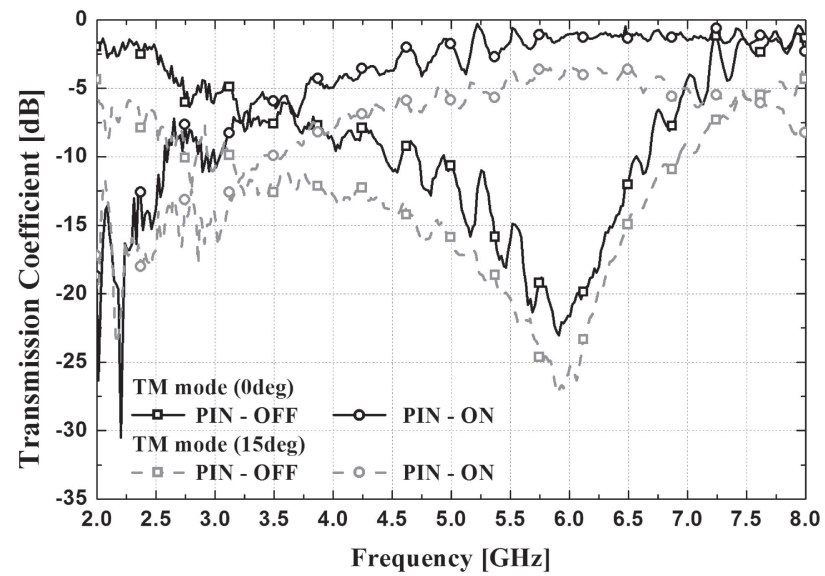

(b) TM mode

Fig. 5. Measured transmission characteristics of the proposed reconfigurable FSS for different incident angles $\left(0^{\circ}\right.$, and $\left.15^{\circ}\right)$ 
$1.3 \%$ was shown in TE mode, and $0.16 \%$ was shown in TM mode. These values show the relative stability of the frequency transmission. Measurements could not be made when the incident angle was $30^{\circ}$ because the jig was too small; thus measurements were made only for cases of $\theta=0^{\circ}$, and $15^{\circ}$. Comparing the simulation results, a resonant frequency error of $3.5 \%$ was shown for a vertical incident wave with an incident angle of $0^{\circ}$ in TE mode. This error is considered to be the result of errors in the inductance and capacitance values of the PIN diode.

\section{Conclusion}

In this paper, a paper-based FSS that can be quickly and inexpensively applied as wallpaper was designed and manufactured using the ink-jet printing method. A PIN diode was used in the proposed FSS, which was designed to block 5-GHz-band signals in the OFF condition and transmit the signals in the ON condition. The designed FSS showed stable characteristics for incident angles and polarization. To verify the operation of the designed FSS, the FSS was printed on a $0.2 \mathrm{~mm}$ thick paper using the ink-jet printing method. An FSS that has a switching function was manufactured by attaching the PIN diode using conductive adhesive. It was confirmed that the measured results corresponded to the calculated results, and that the reconfigured FSS showed stability against polarization and incident angles.

\section{Acknowledgments}

This work was supported by the research grant of the Kongju National University in 2014. 